\title{
A Interação Terapêutica em Intervenções com Universitários com Transtorno de Ansiedade Social
}

\section{Therapeutic Interaction in Interventions with College} Students Suffering from Social Anxiety Disorder

La Interacción Terapéutica en Intervenciones Con los Alumnos Universitarios con Trastorno de Ansiedad Social

\section{Vagner Angelo Garcia, Alessandra} Turini Bolsoni-Silva \& Glaucia Fernanda Galeazzi Nobile

Universidade Estadual Paulista Júlio de Mesquita Filho

http://dx.doi.org/10.1590/1982-3703000842014

(cc) BY 
Resumo: Atualmente, muitas pesquisas têm sido conduzidas para avaliar o papel do terapeuta e do cliente no processo terapêutico. Este trabalho se propôs a analisar sessões de terapia analítico comportamental em intervenções com transtorno de ansiedade social. Vinte e duas sessões de duas díades terapêuticas foram analisadas e categorizadas de acordo com um sistema de categorização de comportamentos (SiMCCIT). Os resultados foram agrupados em frequência e duração nos três momentos da intervenção: Início (primeiras três sessões), Desenvolvimento (cinco sessões intermediárias) e Encerramento (três sessões finais). Os resultados apontam que o papel da terapeuta foi semelhante em ambos os atendimentos, enquanto que os clientes apresentaram algumas diferenças. Houve flexibilidade da terapeuta em adaptar o procedimento de acordo com a necessidade de cada cliente. Foram discutidos aspectos da interação terapêutica que pela análise das categorias permitiu evidenciar uma forma de agir com clientes com transtorno de ansiedade social.

Palavras-chave: Comportamento social. Transtornos fóbicos. Comportamento verbal.

Abstract: Many studies have been conducted to evaluate the roles of the therapist and the client in a therapeutic process. In this segment, this study aimed to analyze the behavioral analytic interventions in therapy sessions with students with social anxiety disorder (SAD). Twenty-two sessions of a therapeutic dyad were analyzed and categorized according to a system of behavior categorization (SiMCCIT). The behavior frequency and duration results were grouped into three stages of intervention: Early Stage (first three sessions), Development Stage (five intermediate sessions), and Closing Stage (final three sessions). Therapist flexibility was present in adapting the procedure according to the needs of each client. Aspects of the therapeutic interaction (highlighted by category analysis) showing the way to act with clients suffering from SAD were discussed.

Keywords: Social behavior. Phobic disorders. Verbal behavior.

Resumen: En la actualidad, se han realizado muchas investigaciones para evaluar la función del terapeuta y del cliente en el proceso terapéutico. Este estudio tuvo como objetivo analizar las sesiones de terapia analíticas conductuales en intervenciones con trastorno de ansiedad social. Fueron analizados y categorizados veintidós sesiones de dos díadas terapéuticas de acuerdo con un sistema de clasificación de los comportamientos (SiMCCIT). Los resultados se agruparon de acuerdo con la frecuencia y la duración de la intervención en los tres momentos: Inicio (las tres primeras sesiones), Desarrollo (cinco sesiones intermedias) y finalización (tres últimas sesiones). Los resultados indican que el papel del terapeuta fue similar en ambas sesiones, mientras que los clientes mostraron algunas diferencias. Hubo flexibilidad del terapeuta para adaptar el procedimiento de acuerdo con las necesidades de cada cliente. Se discutieron aspectos de la interacción terapéutica destacándose, en el análisis de las categorías, la manera de actuar con los clientes con trastorno de ansiedad social.

Palabras clave: Conducta social. Trastornos fóbicos. Conducta verbal.

\section{Introdução}

A análise da interação entre terapeuta e cliente permite levantar dados sobre o que o terapeuta faz na sessão de psicoterapia, bem como quais comportamentos do terapeuta possibilitam resultados sejam eles positivos ou negativos observados no comportamento do cliente (Castonguay \& Beutler, 2006; Meyer, 2001; Meyer \& Vermes 2001). Assim, descrever as sessões de terapia em termos desses comportamentos se constitui um importante ponto de partida para o desenvolvimento da Psicologia Clínica. Essa descrição pode ser feita por meio do registro de áudio e/ou vídeo do comportamento verbal do terapeuta e cliente em termos de categorias 
de comportamentos (Zamignani, 2007; Zamignani \& Meyer, 2007).

O comportamento verbal, segundo Skinner (1957), é um comportamento operante, cuja consequência para o comportamento de quem fala é produzida pelo comportamento de quem ouve. Os operantes verbais são classificados por Skinner (1957) em tato, mando, ecóico, transcritivo (cópia e ditado) e textual, além do autoclítico. Para a classificação do comportamento verbal, leva-se em conta a função do comportamento, isto é, o efeito que provoca sobre o seu ambiente social (Barros, 2003; Matos, 1991).

Assim, os estudos sobre a interação terapêutica consideram para a categorização as ações apontadas pela literatura clínica como típicas de uma interação verbal terapêutica, por exemplo, descrição de eventos, aprovação, inferência e orientação (Zamignani, 2007). Essa análise, segundo Zamignani (2007), permite, por meio da descrição do comportamento verbal vocal, estudar importantes processos da interação clínica, como por exemplo, a tomada de decisão do terapeuta, consequências providas pelo terapeuta às ações do cliente, orientação e aconselhamento, manejo de sentimentos e emoções entre muitos outros. Essa investigação é conhecida como pesquisa de processo, cujo objetivo é identificar os processos de mudança que ocorrem ao longo da interação entre terapeuta e cliente (Zamignani \& Meyer, 2007).

Encontra-se na literatura o termo 'relação terapêutica' como sinônimo de 'interação terapêutica'. Entretanto, Kanamota (2013) defende que 'relação terapêutica' remete a um tipo específico de relação, designado para caracterizar a qualidade dessa relação. Neste trabalho adotaremos apenas o termo interação terapêutica por entender que é objetivo desta pesquisa a descrição de comportamentos do terapeuta e cliente, e não necessariamente avaliar a qualidade dessa relação. Os resultados da análise da interação terapêutica têm reafirmado a complexidade desse relacionamento e a possibilidade de se utilizá-lo na predição dos resultados da terapia (Silveira \& Kerbauy, 2000).
Nesse campo de estudo, diversas pesquisas têm sido conduzidas para avaliar o que o terapeuta faz que proporcione mudanças no cliente. Por exemplo, o estudo conduzido por Falcone, Gil e Ferreira (2007) que avaliaram a frequência de verbalização empática com terapeutas de diferentes abordagens. As sessões foram transcritas e foi solicitado à cliente colaboradora e a três juízes que avaliassem o relato quanto à empatia. Os resultados apontaram diferenças quanto à avaliação dos juízes em relação à avaliação da cliente. Uma das hipóteses levantadas pelos autores é que a cliente realizou sua avaliação sob controle não apenas do relato verbal, mas também pelas manifestações não-verbais dos terapeutas. Diferentemente, os juízes tiveram acesso apenas às transcrições. Os autores apontam essa questão como uma limitação quanto ao estudo da interação terapêutica por observadores apenas por intermédio da transcrição do relato, sem a observação com recurso áudio/visual.

Sobre interação terapêutica, Ruiz-Sancho, Frojan-Parga e Calero-Elvira (2013) tinham como hipótese de pesquisa avaliar de que forma as verbalizações do cliente influenciavam os comportamentos do terapeuta e, para isso, foram analisadas 92 sessões (19 casos clínicos de nove terapeutas especializados em terapia comportamental). O estudo conclui que o terapeuta responde diferencialmente a verbalizações do cliente, modificando as contingências verbais quando o conteúdo das verbalizações do cliente se aproximam ou se tornam mais distantes dos objetivos terapêuticos. Os autores ainda discutem o quanto é importante a condução de estudos sobre a interação terapêutica, pois tais pesquisas buscam auxiliar numa importante lacuna de conhecimento, que aborda sobre o papel do terapeuta na psicoterapia.

Zamignani (2007) se dedicou a desenvolver um Sistema Multidimensional de Categorização de Comportamentos na Interação Terapêutica (SiMCCIT), com base em três eixos. Eixo l: comportamento verbal (vocal e não vocal); Eixo Il: temas da sessão (assunto) e Eixo III: respostas motoras (espreguiçar, bocejar, tamborilar dedos etc). Durante todo 
o processo de desenvolvimento do sistema de categorização, houve inúmeros procedimentos que objetivavam legitimar o estudo, tais como concordância de observadores, treino de categorização entre os observadores e reformulações do sistema. Desde então, pesquisas tem se utilizado do SiMCCIT (Fernandes, 2012; Kameyama 2012; Meyer, 2009; Oshiro, 2011; Sadi, 2011; Silveira, Bolsoni-Silva \& Meyer, 2009; Xavier, 2011).

O estudo conduzido por Silveira et al. (2009) utilizou uma versão preliminar do sistema de categorias proposto por Zamignani (2007), adaptado para o uso em situação terapêutica de grupo. Os dados foram organizados em termos de duração e frequência das categorias. As categorias de terapeuta que tiveram maior frequência e duração foram: Aprovação, Recomendação, Interpretação, Informação e Solicitação de relato, sendo que Recomendação, Informação e Solicitação de reflexão foram apresentadas com frequência maior em relação ao grupo de clientes, já as demais, Aprovação, Interpretação, Solicitação de relato, Reprovação e Empatia, foram apresentadas com frequência maior às clientes individualmente.

Fernandes (2012) buscou investigar a ocorrência de comportamentos de uma terapeuta e sua cliente em sessões iniciais de terapia e também identificar possíveis práticas da terapeuta que contribuíssem para o estabelecimento de uma boa relação terapêutica. A cliente era uma mulher com queixas de depressão e de relacionamento interpessoal. Os resultados apontam que as categorias mais frequentes do comportamento verbal vocal da terapeuta foram de Facilitação $(31,2 \%)$, Empatia $(24,1 \%)$ e Solicitação de relato $(18,1 \%)$, e, para o cliente, a categoria Relato se sobressaiu das demais $(61,1 \%)$. A autora discute que o sistema utilizado permitiu descrever como ocorreu a interação terapeuta-cliente, mas não foi eficaz para detectar comportamentos que favorecessem o estabelecimento da relação terapêutica.

Diversos autores defendem que os resultados da análise da interação terapêutica têm reafirmado a complexidade desse relacionamento e a possibilidade de utilizá-lo na predição dos resultados da terapia (Andrews, 2000; Castonguay, Constantino \& Grosse, 2006; Silveira \& Kerbauy, 2000). Para Tourinho et al. (2007), os dados obtidos pela categorização dos comportamentos da interação terapeutacliente podem ser analisados juntamente a outras variáveis do processo terapêutico (medidas de resultado, informações sobre o atendimento), possibilitando uma interpretação e discussão mais criteriosa.

Descrever e analisar a interação terapêutica são características das pesquisas de processo, nas quais se busca caracterizar a interação (verbal e não verbal) entre terapeuta e cliente com o intuito de identificar os processos de mudança que permeiam essa interação (Zamignani \& Meyer, 2011). Nesse sentido, uma questão que se coloca é se existe uma relação entre o tipo de interação com o tipo de queixa e o resultado da psicoterapia. Para responder a essa pergunta, é preciso delimitar sobre qual população será estudada a interação terapêutica e, neste caso, trata-se do atendimento a universitários com transtorno de ansiedade social.

Transtorno de Ansiedade Social (TAS) e Interação Terapêutica

O transtorno de ansiedade social apresenta como característica marcante um padrão de comportamento evitativo de situações sociais ocasionando prejuízos na vida profissional, acadêmica e social do indivíduo (Falcone, 1995; Rocha, 2012). Caracteriza-se pelo medo excessivo, persistente e irracional de uma ou mais situações nas quais o indivíduo é exposto à possível avaliação por parte de outros (por exemplo, comer, escrever, falar em público, interagir com o sexo oposto), temendo comportar-se de maneira humilhante, embaraçosa e/ou a desaprovação ou rejeição por parte dos pares (APA, 2013). Sua prevalência em seis meses é de que a cada 100 pessoas, de 2 a 3 apresentam critérios para o diagnóstico, sendo os sinais apresentados dos 5 aos 35 anos de idade, com ápice na adolescência (Kaplan, Sadock \& Grebb, 1997).

Recentemente lançado nos Estados Unidos, o Manual Diagnóstico e Estatístico de 
1 Banco de dados que, na área de Psicologia, contempla 38 bases, compreendendo as principais fontes de pesquisa na área, como por exemplo, Scielo, Pepsic, PsyclNFO (APA), Scopus, Web of Science. Pesquisa realizada no mês de dezembro de 2013.

2 Naquela ocasião denominada de fobia social.
Transtornos Mentais/DSM-V (APA, 2013) apresenta algumas diferenças do DSM-IV-TR (APA, 2002) em relação à caracterização do transtorno de ansiedade social. As principais diferenças são: em relação à nomenclatura, em que a fobia social passa a ser denominada simplesmente de Transtorno de Ansiedade Social (TAS); e, em relação a sintomatologia e diagnóstico, os sintomas devem ter duração mínima de seis meses ou mais (antes o prazo era requisito apenas para diagnóstico em crianças). Assim, esse período mínimo de presença dos sintomas reduz a possibilidade de que um indivíduo esteja vivenciando apenas um medo temporário/transitório.

Há uma carência de estudos sobre a interação terapêutica em intervenções com clientes com ansiedade social, sobretudo na perspectiva analítico-comportamental. Essa lacuna foi comprovada após pesquisa no banco de dados da Capes ${ }^{1}$, com dois conjuntos de palavras chaves: conjunto A (therapeutic interaction; therapeutic relationship) e conjunto B (social anxiety disorder; social phobia), em que deveria aparecer no assunto pelo menos uma palavra de cada conjunto em artigos científicos de qualquer nacionalidade e ano, e não foram encontrados estudos que contemplassem os termos propostos. Descrever o que faz o terapeuta numa intervenção com clientes com ansiedade social, ou seja, descrever a interação terapêutica desse processo permitiria que psicólogos e outros profissionais avaliassem as conclusões e resultados apresentados e a partir disso determinar quais estratégias e intervenções produzem com maior probabilidade os efeitos pretendidos e, então, adaptá-las a populações específicas.

Este trabalho se propôs a analisar a interação terapêutica em intervenções com universitários com o transtorno de ansiedade social. A intervenção é descrita em Rocha (2012), que se propõem a avaliar a eficácia dos efeitos de uma intervenção comportamental com treinamento em habilidades sociais sobre o repertório de estudantes universitários que apresentavam ansiedade social ${ }^{2}$. As autoras avaliaram as habilidades sociais e o diagnóstico do transtorno dos participantes antes da intervenção, após, e em seguimento (quatro meses depois). Foram utilizados tanto medidas de produto quanto de processo, além de vídeos dessa intervenção, que é considerada de sucesso por ter atingido seus objetivos, ocorrendo a remissão do transtorno.

O objetivo deste trabalho é descrever, em termos de frequência e duração, os comportamentos do terapeuta e do cliente presentes nas sessões de terapia comportamental no atendimento com universitários com transtorno de ansiedade social e comparar se houve interações diferentes entre cada díade terapêutica em relação aos dados de frequência.

\section{Método}

\section{Participantes}

Participaram da pesquisa dois universitários diagnosticados com transtorno de ansiedade social, sem comorbidades, que passaram por um procedimento (individual) de intervenção comportamental que inclui treino de habilidades sociais (Rocha, 2012) e uma terapeuta com três anos de experiência. $\mathrm{O}$ Participante 1 (P1), do sexo masculino, com 19 anos, cursava o $2^{\circ}$ ano de bacharelado em Ciência da Computação, solteiro/sem namorada, não trabalhava e residia em república com um amigo. O Participante 2 (P2), do sexo feminino, com 22 anos, cursava o $4^{\circ}$ ano de Pedagogia, solteira com namorado, não trabalhava, mas realizava estágio curricular, morando em uma república com quatro amigas.

O projeto de pesquisa foi aprovado pelo Comitê de Ética da universidade ao qual estava vinculado. As sessões foram gravadas em vídeo, sendo que clientes e terapeuta assinaram um Termo de Consentimento Livre e Esclarecido (TCLE) autorizando a utilização das filmagens. As sessões foram conduzidas num Centro de Psicologia Aplicada de uma Universidade do interior do Estado de São Paulo. Os clientes P1 e P2 passaram por todo o procedimento (treinamento em habilidades sociais e terapia 
30 procedimento de intervenção foi aplicado em 12 sessões para cada participante, contudo, foram analisadas apenas 11 sessões de cada participante, pois ocorreram problemas em dois arquivos de vídeo: sessão 7 do P1 e sessão 10 do P2. comportamental), concluindo 12 sessões cada (Rocha, 2012). Ambos os participantes apresentaram ganhos comportamentais, aumentando o repertório de comportamentos socialmente habilidosos, e, em relação ao transtorno, tanto após a intervenção quanto em seguimento, os participantes deixaram de apresentar o diagnóstico de ansiedade social. Isso possibilitou aos participantes uma melhor adaptação à universidade bem como interações mais saudáveis tanto no contexto pessoal quanto profissional. Maiores detalhes do procedimento em Rocha (2012).

\section{Material}

Neste trabalho optou-se pela análise da interação terapêutica por meio do recurso audiovisual (áudio e vídeo), pois, como apontado por Falcone et al. (2007), esse recurso auxilia o observador na categorização da sessão. Assim, foram utilizados arquivos de vídeo que continham as intervenções ( 11 sessões para cada participante ${ }^{3}$ ) totalizando $38 \mathrm{~h} 15 \mathrm{~min}$, sendo o tempo de cada sessão variando de 50 até $120 \mathrm{~min}$.

Para o estudo das categorias, foi utilizado o Protocolo de Observação (Zamignani, 2007), que continha as definições das categorias comportamentais relacionadas ao Eixo I: comportamento verbal vocal e não vocal, do Terapeuta ( 15 categorias) e do Cliente (13 categorias). Também foi utilizado o software $\mathrm{Clic}^{\circledR}$ (desenvolvido por Zamignani para o treino de observadores quanto ao correto uso das categorias) e o software The Observer XT 7.0 para a categorização das sessões, além do programa estatístico SPSS (Versão 17.0) para análise dos dados.

Procedimento de tratamento e análise dos dados

A análise das sessões foi realizada pelo pesquisador e mais um observador. A pesquisa foi desenvolvida em cinco fases conforme descritas, a seguir:

Fase I - Estudo e Treino quanto ao uso do SiMCCIT (Zamignani, 2007).
Fase II - Consenso entre Observadores: após o estudo e o treino, inicia-se esta fase com o objetivo de avaliar o grau de concordância entre os observadores (Kazdin, 1982). Essa concordância foi avaliada através de uma ferramenta do software The Observer XT 7.0 que permite uma medida do percentual de concordância entre os observadores, bem como o índice do coeficiente kappa.

Nesta fase II analisou-se 30 minutos de uma sessão escolhida aleatoriamente, com o objetivo de verificar se o índice se encontrava dentro do satisfatório: percentual de concordância acima de 70\% (Fagundes, 1999) e coeficiente kappa acima de 0,60 (Fonseca et al., 2007). Enquanto o índice não estivesse satisfatório, novo estudo era realizado pelos observadores com treino nas categorias em que a concordância encontrava-se baixa, repetindo-se o procedimento inicial até a obtenção de índices de concordância satisfatório. Assim, foram categorizados 30 min de uma sessão escolhida aleatoriamente. Os resultados demonstraram divergências entre os observadores, com um índice nada satisfatório de $51 \%$ e coeficiente Kappa 0,49. Após passarem novamente pelo procedimento da Fase I, foram categorizados outros 30 min desta mesma sessão, porém, o percentual de concordância ainda ficou insatisfatório, com $66 \%$ de concordância e o coeficiente Kappa 0,62. Novamente os observadores refizeram o Treino no software $\mathrm{Clic}^{\circledR} \mathrm{e}$ discutiram sobre o sistema de categorização, com ênfase nas categorias que apresentavam maiores divergências. Neste terceiro período de categorização, foi atingido o patamar satisfatório, com $87 \%$ de concordância, sendo o coeficiente Kappa 0,86. Uma vez atingido este percentual, os observadores prosseguiram para a próxima fase para obtenção do índice de concordância final.

Fase III - Concordância entre observadores: foram sorteados aleatoriamente $20 \%$ do total de sessões para análise $(4,4$ sessões, aproximado para cinco sessões), com o objetivo de avaliar o índice de concordância entre os observadores com relação ao uso do SiMCCIT (Zamignani, 2007). Essa fase difere da anterior uma vez que não há mais comunicação entre os observadores. 
As sessões sorteadas e os respectivos índices de concordância obtidos foram: $\mathrm{P} 1$ sessão 01 : Concordância 81\%, Coeficiente Kappa 0,79; P1 sessão 10: Concordância 79\%, Coeficiente Kappa 0,78; P1 sessão 11: Concordância $81 \%$, Coeficiente Kappa 0,80; P2 sessão 03: Concordância 81\%, Coeficiente Kappa 0,80 P2 sessão 12: Concordância 85\%, Coeficiente Kappa 0,84. Como podemos observar, os índices se mantiveram num patamar satisfatório, acima de $70 \%$ (Fagundes, 1999) e coeficiente kappa acima de 0,60 (Fonseca et al., 2007), e mantiveram regularidade entre as sessões.

Fase IV - Categorização: terminada a fase de concordância, as sessões faltantes foram divididas entre os observadores para categorização.

Fase $V$ - Análise e interpretação dos resultados: após a categorização das sessões faltantes (Fase IV), os dados da categorização foram exportados para o programa SPSS e agrupados de acordo com o momento do processo terapêutico: I) Início da Terapia (três sessões iniciais), II) Desenvolvimento da terapia (cinco sessões intermediárias), e III) Encerramento da Terapia (três sessões finais). Em cada um desses períodos foi realizada a média aritmética simples e a respectiva porcentagem das categorias de comportamentos, obtendo assim um índice que permitiria uma comparação entre cada momento da intervenção: início, desenvolvimento e encerramento da terapia. Para comparar se os resultados encontrados para um cliente se replicavam em outro, foi realizado o teste estatístico $U$ de Mann-Whitney com os dados de frequência.

\section{Resultados}

Os resultados, a seguir, apresentam os dados organizados em frequência e duração. A Tabela 1 apresenta as médias de frequência e de porcentagem das categorias de respostas emitidas pelo terapeuta conforme a divisão em início/desenvolvimento/encerramento, em relação ao P1 e ao P2. A análise da frequência de comportamentos tem por objetivo evidenciar os padrões de respostas de terapeuta e cliente para o processo terapêutico (Xavier,
2011). A porcentagem foi calculada em cada fase do atendimento (Início, Desenvolvimento e Encerramento) tanto em relação aos dados de frequência (Tabela 1) quanto de duração (Tabela 2) através da multiplicação por 100 da média simples do período de cada categoria, sendo o total dividido pela soma de categorias do período.

Podem-se destacar as categorias em três grupos de acordo com a sua frequência: com alta média de frequência (acima de $10 \%$ ), os de média intermediária (entre 3 e $10 \%$ ) e os com baixa média próximas (abaixo de 3\%). No primeiro grupo, há uma categoria que se destaca das demais, com grande expressividade, para ambos os clientes: Gestos de concordância T. Outra categoria com alta frequência foi a de Facilitação. A categoria Solicita relato apresentou frequência estável para o P2 e apenas durante o encerramento esteve abaixo dessa estabilidade para o P1.

No segundo grupo, as categorias com média de frequência entre 3 e 10\%, foram: Solicita Reflexão, Informações, Interpretação, Recomendação, Aprovação e Empatia. Observa-se que Solicita Reflexão foi maior durante o desenvolvimento da terapia para P1 e estável para P2. Informações foi maior durante o início da terapia para P1, decrescendo com o avanço do tratamento e estável para P2. Interpretação ficou estável para P1 e manteve-se crescente para P2. Recomendação e Empatia não apresentaram variações significativas e Aprovação foi aumentando de média durante o avanço da terapia.

O terceiro grupo, com taxas de frequência próximas a zero, contemplam uma categoria residual (Insuficiente $\mathrm{T}$ - dificuldade em compreender a fala do terapeuta), categorias de Gestos de comando, Gestos outros e de Gestos de discordância do terapeuta, Reprovação.

A Tabela 2 apresenta a média de duração das categorias em segundos. Observa-se que diferentemente das médias da frequência, a categoria com maior duração (média) foi a de Informações, seguidas de Interpretação 
Tabela 1. Média de Frequência e Porcentagem de Respostas Emitidas pelo Terapeuta

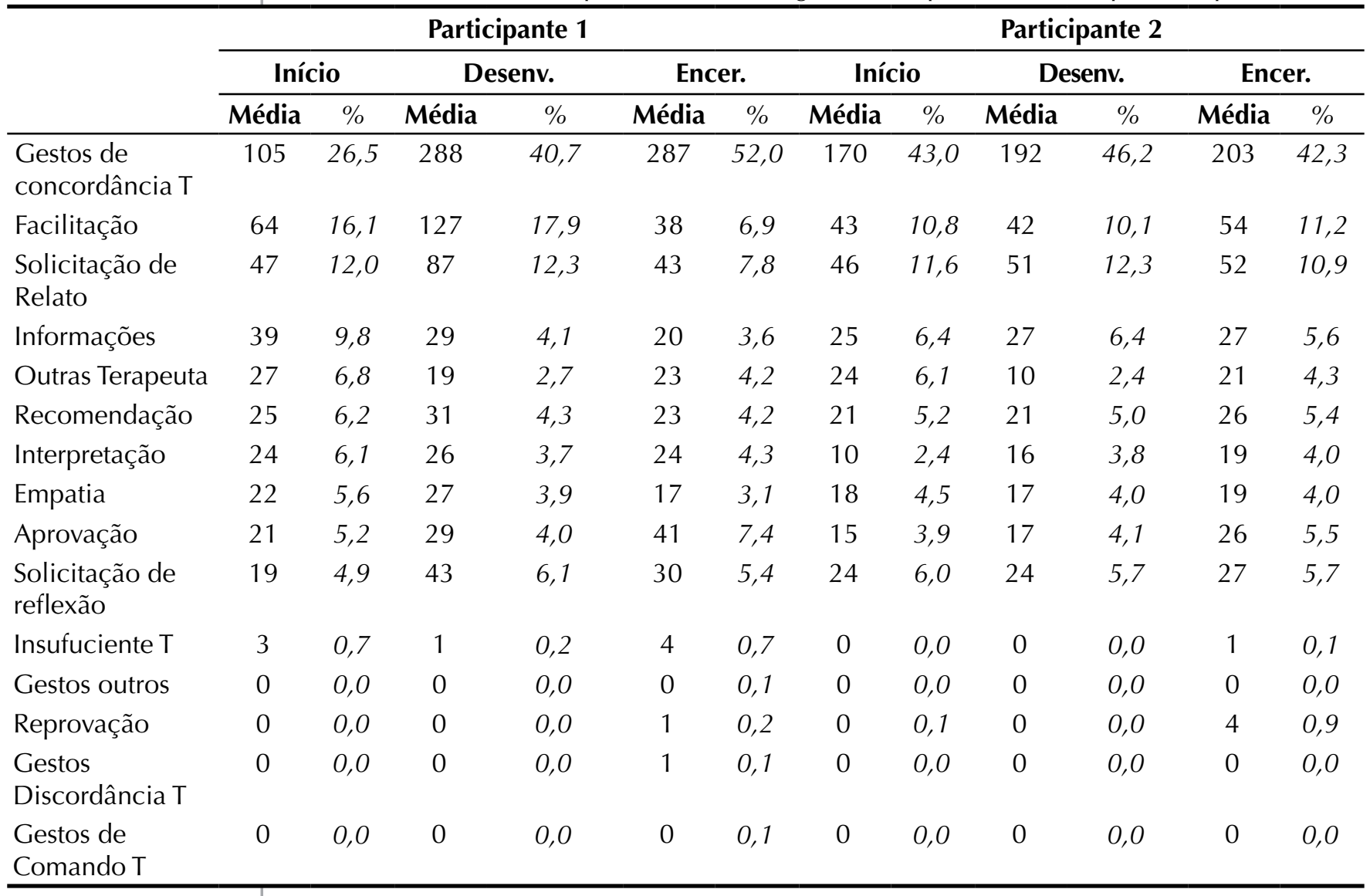

Tabela 2. Média de Duração (Segundos) e Porcentagem das Respostas Emitidas peloTerapeuta*

Participante 1

Participante 2

\begin{tabular}{|c|c|c|c|c|c|c|c|c|c|c|c|c|}
\hline & \multicolumn{2}{|c|}{ Início } & \multicolumn{2}{|c|}{ Desenv. } & \multicolumn{2}{|c|}{ Encer. } & \multicolumn{2}{|c|}{ Início } & \multicolumn{2}{|c|}{ Desenv. } & \multicolumn{2}{|c|}{ Encer. } \\
\hline & Média & $\%$ & Média & $\%$ & Média & $\%$ & Média & $\%$ & Média & $\%$ & Média & $\%$ \\
\hline Informações & 704 & 34,1 & 640 & 26,7 & 385 & 19,8 & 766 & 34,0 & 664 & 34,1 & 459 & 23,5 \\
\hline Interpretação & 640 & 20,7 & 407 & 17,0 & 445 & 22,9 & 279 & 12,4 & 3,9 & 15,9 & 327 & 16,7 \\
\hline Recomendeção & 184 & 8,9 & 344 & 14,3 & 198 & 10,2 & 278 & 12,3 & 204 & 10,5 & 298 & 15,2 \\
\hline $\begin{array}{l}\text { Solcitação de } \\
\text { Relato }\end{array}$ & 180 & 8,7 & 275 & 11,5 & 135 & 7,0 & 241 & 10,7 & 200 & 10,2 & 159 & 8,1 \\
\hline Aprovação & 177 & 8,6 & 286 & 11,9 & 243 & 12,5 & 172 & 7,6 & 159 & 8,2 & 282 & 14,4 \\
\hline $\begin{array}{l}\text { Solicitação de } \\
\text { Reflexão }\end{array}$ & 161 & 7,8 & 186 & 7,8 & 155 & 8,0 & 171 & 7,6 & 137 & 7,0 & 135 & 6,9 \\
\hline Outras Terapeuta & 120 & 5,8 & 99 & 4,1 & 243 & 12,5 & 225 & 10,0 & 128 & 6,6 & 117 & 6,0 \\
\hline Empatia & 110 & 5,3 & 159 & 6,6 & 127 & 6,5 & 121 & 5,4 & 146 & 7,5 & 155 & 7,9 \\
\hline Insfuciciente T & 3 & 0,1 & 1 & 0,1 & 7 & 0,4 & 0 & 0,0 & 0 & 0,0 & 2 & 0,1 \\
\hline Reprovação & 0 & 0,0 & 0 & 0,0 & 4 & 0,2 & 1 & 0,0 & 0 & 0,0 & 23 & 1,2 \\
\hline
\end{tabular}

*Facilitação, Gestos de Concordância Terapeuta, Gestos Discordância T, Gestos de Comando T e Gestos Outros são categorias tipo evento, e não apresentam duração, portanto foram retiradas da tabela. 
e Recomendação. Outro ponto interessante é que, na medida em que a terapia avançava, diminuía o tempo dispensado a Informações pelo terapeuta, esse dado é relevante para ambos os clientes. Sobre o comportamento de Recomendação, o terapeuta o fez com maior tempo no período de desenvolvimento para o P1, e no período de encerramento para o P2.

A Tabela 3 apresenta a média da frequência das categorias do cliente. Observamos que a categoria com a maior média de respostas foi a categoria não vocal de Gestos de concordância C. Neste caso, observemos uma diferença considerável entre P1 e P2. Por sua vez, a categoria de Concordância (respostas vocais) manteve-se estável para ambos os clientes do início ao desenvolvimento da terapia e aumento para o encerramento. Entretanto, manteve-se a diferença significativa entre os clientes, pois o P1 apresentava até seis vezes mais Concordância (vocal) do que o P2 nas fases iniciais da terapia, e três vezes mais no encerramento.

A categoria de Relato apresentou maior frequência durante o desenvolvimento. A categoria de Estabelecer relações para o P1 foi mais longa durante o desenvolvimento e para P2 foi crescente com o avanço da terapia. A categoria de Solicitação apresentou ligeiro decréscimo com o avanço da terapia para ambos os clientes. A categoria de Melhora foi estável para P1, crescente para P2, e Metas foi crescente para ambos, com maior destaque para P1. Assim como nas categorias do Terapeuta, ambos os participantes não apresentaram comportamentos de Oposição e Discordância Cliente.

Em relação à duração das categorias do cliente, a Tabela 4 apresenta as médias nos três momentos da terapia. A categoria com maior média foi Relato e, assim como em relação à frequência, houve maior tempo de

Tabela 3. Média de Frequência e Porcentagem de Respostas Emitidas pelo Cliente

\begin{tabular}{|c|c|c|c|c|c|c|c|c|c|c|c|c|}
\hline & \multicolumn{6}{|c|}{ Participante 1} & \multicolumn{6}{|c|}{ Participante 2} \\
\hline & \multicolumn{2}{|c|}{ Início } & \multicolumn{2}{|c|}{ Desenv. } & \multicolumn{2}{|c|}{ Encer. } & \multicolumn{2}{|c|}{ Início } & \multicolumn{2}{|c|}{ Desenv. } & \multicolumn{2}{|c|}{ Encer. } \\
\hline & Média & $\%$ & Média & $\%$ & Média & $\%$ & Média & $\%$ & Média & $\%$ & Média & $\%$ \\
\hline $\begin{array}{l}\text { Gestos de } \\
\text { concordância C }\end{array}$ & 132 & 39,4 & 130 & 41,2 & 143 & 37,1 & 44 & 25,9 & 44 & 25,9 & 61 & 27,5 \\
\hline $\begin{array}{l}\text { Concordância } \\
\text { (vocal) }\end{array}$ & 71 & 21,2 & 73 & 15,9 & 90 & 23,3 & 11 & 6,6 & 11 & 6,6 & 27 & 12,3 \\
\hline Relato & 68 & 20,4 & 107 & 23,3 & 75 & 19,4 & 55 & 31,9 & 64 & 37,4 & 57 & 25,9 \\
\hline $\begin{array}{l}\text { Estabelece } \\
\text { Relações }\end{array}$ & 21 & 6,4 & 44 & 9,5 & 35 & 9,1 & 21 & 12,3 & 26 & 15,5 & 31 & 14,2 \\
\hline Outras Cliente & 19 & 5,8 & 24 & 5,2 & 17 & 4,4 & 26 & 15,4 & 9 & 5,5 & 27 & 12,3 \\
\hline Solicitação & 14 & 4,3 & 12 & 2,6 & 9 & 2,4 & 10 & 5,6 & 10 & 5,6 & 8 & 3,5 \\
\hline $\begin{array}{l}\text { Insuficiente } \\
\text { Cliente }\end{array}$ & 4 & 1,1 & 2 & 0,5 & 5 & 1,4 & 1 & 0,4 & 0 & 0,2 & 2 & 0,8 \\
\hline Melhora & 3 & 0,9 & 4 & 0,9 & 4 & 1,0 & 0 & 0,0 & 1 & 0,6 & 3 & 1,4 \\
\hline Metas & 2 & 0,5 & 3 & 0,7 & 6 & 1,5 & 1 & 0,8 & 2 & 1,2 & 2 & 0,9 \\
\hline Gestos Outros C & 0 & 0,1 & 0 & 0,0 & 1 & 0,3 & 2 & 1,2 & 2 & 1,4 & 3 & 1,2 \\
\hline Oposição & 0 & 0,0 & 0 & 0,0 & 0 & 0,1 & 0 & 0,0 & 0 & 0,2 & 0 & 0,0 \\
\hline $\begin{array}{l}\text { Gestos } \\
\text { comando C }\end{array}$ & 0 & 0,0 & 0 & 0,0 & 0 & 0,0 & 0 & 0,0 & 0 & 0,0 & 0 & 0,0 \\
\hline $\begin{array}{l}\text { Gestos de } \\
\text { discordância C }\end{array}$ & 0 & 0,0 & 0 & 0,0 & 0 & 0,0 & 0 & 0,0 & 0 & 0,0 & 0 & 0,2 \\
\hline
\end{tabular}


Tabela 4. Média de Duração (Segundos) e de Porcentagem das Respostas Emitidas pelo Cliente*

\begin{tabular}{|c|c|c|c|c|c|c|c|c|c|c|c|c|}
\hline & \multicolumn{6}{|c|}{ Participante 1} & \multicolumn{6}{|c|}{ Participante 2} \\
\hline & \multicolumn{2}{|c|}{ Início } & \multicolumn{2}{|c|}{ Desenv. } & \multicolumn{2}{|c|}{ Encer. } & \multicolumn{2}{|c|}{ Início } & \multicolumn{2}{|c|}{ Desenv. } & \multicolumn{2}{|c|}{ Encer. } \\
\hline & Média & $\%$ & Média & $\%$ & Média & $\%$ & Média & $\%$ & Média & $\%$ & Média & $\%$ \\
\hline Relato & 1166 & 62,9 & 2027 & 64,4 & 1958 & 61,9 & 1365 & 57,4 & 1997 & 63,5 & 1822 & 55,7 \\
\hline $\begin{array}{l}\text { Estabelece } \\
\text { Relações }\end{array}$ & 426 & 22,9 & 699 & 22,2 & 645 & 20,4 & 683 & 28,7 & 841 & 26,7 & 850 & 25,8 \\
\hline $\begin{array}{l}\text { Concordância } \\
\text { (vocal) }\end{array}$ & 98 & 5,3 & 124 & 3,9 & 143 & 4,5 & 17 & 0,7 & 36 & 1,1 & 47 & 1,4 \\
\hline Outras Cliente & 67 & 3,6 & 144 & 4,6 & 293 & 9,3 & 247 & 10,4 & 163 & 5,2 & 338 & 10,3 \\
\hline Solicitação & 52 & 2,8 & 43 & 1,4 & 22 & 0,7 & 51 & 2,1 & 49 & 1,6 & 43 & 1,3 \\
\hline Melhora & 29 & 1,6 & 74 & 2,3 & 59 & 1,9 & 0 & 0,0 & 13 & 0,4 & 112 & 3,4 \\
\hline Metas & 12 & 0,6 & 30 & 1,0 & 35 & 1,1 & 15 & 0,6 & 38 & 12 & 77 & 2,3 \\
\hline $\begin{array}{l}\text { Insuficinete } \\
\text { Cliente }\end{array}$ & 6 & 0,3 & 3 & 0,1 & 8 & 0,2 & 1 & 0,0 & 0 & 0,0 & 4 & 0,1 \\
\hline Oposição & 0 & 0,0 & 1 & 0,0 & 1 & 0,0 & 0 & 0,0 & 9 & 0,3 & 0 & 0,0 \\
\hline
\end{tabular}

*Gestos de concordância C, Gestos de discordância C, Gestos de comando C, Gestos outros C, são categorias tipo evento, e não apresentam duração, portanto foram retiradas da tabela.

Relato durante a fase de desenvolvimento da terapia para ambos os clientes.

A categoria Estabelece Relações se destacou das demais, por apresentar também um alto tempo médio de duração. A duração da categoria Solicitação apresentou um decréscimo em seu tempo médio de exposição, assim como nas taxas de frequência. As categorias que evidenciam a melhora do cliente (Melhora) e a formulação de metas (Metas) apresentaram aumento substancial durante o desenvolvimento da terapia.

A Tabela 5 apresenta as médias e valor de $p$ de acordo com o Teste $U$ de Mann-Whitney para as categorias do terapeuta e cliente. Esse teste analisa as diferenças entre as médias e se essa diferença tem significância estatística. Observa-se que para as categorias do terapeuta: Facilitação, Terapeuta Registro Insuficiente e Interpretação são estatisticamente significantes $(p<0,05)$. Isso quer dizer que essas categorias diferem entre si enquanto de um cliente para outro. O mesmo ocorre com cinco das treze categorias do cliente: Relato, Melhora, Concordância C (vocal), Gestos de Concordância (não vocal) e Gestos outros cliente.

\section{Discussão}

Para Oshiro (2011) e Sadi (2011), é importante comparar duas dimensões do comportamento do terapeuta: frequência e duração. Utilizar somente a frequência seria um risco, pois colocaria em comparação categorias distintas, como por exemplo, Facilitação caracterizada por verbalizações mínimas com outras categorias de maior duração, como Informações e Interpretação (Zamignani, 2007; Zamignani \& Meyer, 2009).

De acordo com as Tabelas 1 e 2, categorias de repostas do terapeuta, podemos elencar um primeiro conjunto de categorias, com alta frequência (Facilitação e Gestos de Concordância T). Os resultados aqui encontrados divergem dos achados de Pergher e Negrão (2012) que, analisando a interação terapêutica em uma intervenção com foco no desenvolvimento de comportamentos pró-estudo, encontraram como categoria mais frequente a Solicitação de Relato. Esse dado mostra que o terapeuta age de forma diferente de acordo com o cliente, diagnóstico e tipo de intervenção.

Uma característica da intervenção aqui analisada (terapia comportamental e treinamento em 
Tabela 5. Média e Valor de $p$ de Acordo com o Teste $U$ de Mann-Whitney das Categorias de Respostas do Terapeuta e Cliente (Dados de Frequência)*

\begin{tabular}{lccccccc}
\hline Categorias do terapeuta & $\begin{array}{c}\text { Média } \\
\text { P1 }\end{array}$ & Média P2 & Valor de $\boldsymbol{p}$ & Categorias do cliente & $\begin{array}{c}\text { Média } \\
\text { P1 }\end{array}$ & $\begin{array}{c}\text { Média } \\
\text { P2 }\end{array}$ & $\begin{array}{c}\text { Valor } \\
\text { de } \boldsymbol{p}\end{array}$ \\
\hline Aprovação & 29,7 & 19,2 & 0,114 & Concordância (vocal) & $\mathbf{7 7 , 1}$ & $\mathbf{1 5 , 6}$ & $\mathbf{0 , 0 1 0}$ \\
Empatia & 23,3 & 17,6 & 0,669 & Estabelece Relações & 35,3 & 26,3 & 0,250 \\
Facilitação & $\mathbf{8 5 , 4}$ & $\mathbf{4 5 , 3}$ & $\mathbf{0 , 0 3 5}$ & Gestos de comando C & 0,1 & 0,0 & 0,317 \\
Gestos de Comando T & 0,1 & 0,0 & 0,317 & Gestos de concordância C & $\mathbf{1 6 1 , 4}$ & $\mathbf{4 8 , 7}$ & $\mathbf{0 , 0 0 1}$ \\
Gestos de concordância T & 237,7 & 188,8 & 0,450 & Gestos de discordância C & 0,0 & 0,1 & 0,317 \\
Gestos de discordância T & 0,2 & 0,0 & 0,317 & Gestos Outros C & $\mathbf{0 , 5}$ & $\mathbf{2 , 4}$ & $\mathbf{0 , 0 3 1}$ \\
Gestos outros & 0,2 & 0,0 & 0,147 & Insuficente cliente & 3,6 & 0,8 & 0,196 \\
Informações & 2,2 & 26,3 & 0,793 & Melhora & $\mathbf{3 , 7}$ & $\mathbf{1 , 3}$ & $\mathbf{0 , 0 2 1}$ \\
Insuficiente T & $\mathbf{2 , 4}$ & $\mathbf{0 , 3}$ & $\mathbf{0 , 0 4 7}$ & Metas & 3,6 & 1,8 & 0,133 \\
Interpretação & $\mathbf{2 5 , 1}$ & $\mathbf{1 4 , 9}$ & $\mathbf{0 , 0 3 8}$ & Oposição & 0,2 & 0,2 & 1,000 \\
Outras terapeuta & 22,4 & 16,7 & 0,358 & Outras Cliente & 20,8 & 18,8 & 0,374 \\
Recomendação & 27,0 & 22,1 & 0,598 & Relato & $\mathbf{8 7 , 8}$ & $\mathbf{5 9 , 5}$ & $\mathbf{0 , 0 2 0}$ \\
Reprovação & 0,4 & 1,3 & 0,961 & Solicitação & 12,0 & 9,1 & 0,449 \\
Solicitação de Reflexão & 33,0 & 24,7 & 0,237 & & & & \\
Solicitação de Relato & 64,2 & 50,0 & 0,293 & & &
\end{tabular}

habilidades sociais para clientes com ansiedade social), é que durante todo o seu processo (para ambos os clientes), a terapeuta apresentou médias consideráveis para o comportamento de estimular o relato do cliente (Solicita Relato), e também facilitar esse relato (Facilitação e Gestos de ConcordânciaT). Nesse sentido, Rossi (2012) defende que ouvir o que o cliente tem a dizer contribui para o estabelecimento de um bom vínculo terapêutico.

Dados semelhantes foram encontrados por Fernandes (2012) que, analisando cinco sessões iniciais de terapia, encontrou como categoria mais frequente a Facilitação (31,82\%). Oshiro (2011) também encontrou a Facilitação com maior frequência para ambos os clientes $(37,82 \%$ e $32,26 \%$ ). Observando o participante da pesquisa de Fernandes (2012) - mulher com depressão e queixa de relacionamento interpessoal - e os participantes da pesquisa de Oshiro (2011) - clientes difíceis, com queixa de relacionamento interpessoal-, observa-se uma relação entre o diagnóstico deles com a intervenção aqui analisada: todos os participantes apresentavam problemas de relacionamento interpessoal. Essa semelhança entre as queixas dos clientes, analisadas em conjunto com as frequências das categorias da interação terapêutica sugerem que no caso de clientes com dificuldades de relacionamento interpessoal, a facilitação do relato do cliente é uma estratégia utilizada e bem-sucedida.

O segundo conjunto de categorias envolvia os comportamentos de Solicitação de Reflexão, Informações, Interpretação, Recomendação, Aprovação e Empatia. Dessas, as frequências apresentaram variações para ambos os clientes, mas com médias próximas.

Em relação à categoria Informações, observa-se que, para o P1, houve um decréscimo gradual na média de frequência e tempo dessa categoria, enquanto que para o P2 Informações se manteve estável em frequência e decrescente em tempo. Fernandes (2012) encontrou uma média muito menor da frequência para Informações (1,09\%). Essa divergência pode 
ser explicada quando observamos o procedimento da intervenção utilizado (Rocha, 2012), que associava Terapia Comportamental com um Treino em Habilidades Sociais. Utilizando uma cartilha com temas pertinentes (Bolsoni-Silva, 2009), a terapeuta informava sobre habilidades sociais, justificando o elevado tempo médio (Tabela 2 ) da categoria Informações, uma vez que informar sobre habilidades de comunicação, comportamento habilidoso era uma prerrogativa da terapia.

Em relação à categoria Interpretação, observa-se que a terapeuta passou a fazer mais interpretações ao longo da intervenção para o P2 e se manteve estável para o P1. Essa diferença encontrada para a categoria Interpretação também foi detectada pelo Teste $U$ de Mann-Whitney (Tabela 5), evidenciando que a terapeuta divergiu significativamente na condução da intervenção no que se refere a essa categoria.

Uma explicação para a diferença em relação à categoria comportamental de Interpretação encontrada nos resultados pode estar associada com as queixas dos participantes. O P2, segundo Rocha (2012), conhecia o comportamento habilidoso para a situação, mas não o colocava em prática, necessitando assim de maior esclarecimento por parte da terapeuta, característico da categoria Interpretação, que contempla verbalizações nas quais o terapeuta descreve, supõe ou infere relações causais e/ou explicativas (funcionais, correlacionais, ou de contiguidade) a respeito do comportamento do cliente/terceiros, ou identifica padrões de interação (Zamignani, 2007).

Assim, as diferenças encontradas entre os clientes, mais Informações para P1 e menos para P2, e mais Interpretação para P2 e menos para $\mathrm{P} 1$, são justificados analisando os estudos de caso, onde P1 não sabia como se expressar de maneira habilidosa (por isso mais Informações), e P2 sabia a forma correta de se expressar, mas não agia dessa forma (por isso mais Interpretação). Essa flexibilização é prevista no procedimento de intervenção descrito por Bolsoni-Silva (2009), em que. apesar de ser semiestruturado, as sessões são conduzidas de acordo com as necessidades de cada cliente, levantadas previamente num estudo de caso e reavaliadas constantemente durante a condução da terapia.

A categoria Aprovação apresentou crescimento constante do início da terapia para seu término, em ambos os clientes, e comparando essas médias com os resultados apresentados por Rocha (2012), observamos que ao longo das sessões os clientes passaram a atuar de forma mais habilidosa, arriscando-se mais nas interações, o que proporcionou a Terapeuta mais Aprovações, uma vez que por meio deste comportamento o Terapeuta pode selecionar e fortalecer aspectos do comportamento do cliente (Meyer, 2009).

Em relação às categorias comportamentais de respostas emitidas pelo cliente, observa-se pela Tabela 5 que houve uma divergência na forma com que cada cliente se comportou em sessão, o que demonstra a particularidade de cada um, mesmo sendo uma única terapeuta e o mesmo procedimento de intervenção aplicado. A categoria de respostas não vocais de Gestos de concordância do cliente se destacou para o participante $1 \mathrm{com}$ mais frequência, ficando acima dos $37 \%$ em todos os momentos da intervenção, diferentemente do P2 em que tal categoria ficou entre $25 \mathrm{e}$ $27 \%$, tendo a categoria de Relato como mais frequente. Nesse caso, observa-se a grande diferença entre P1 e P2. Observando o estudo de caso descrito em Rocha (2012), constata-se que um dos objetivos comportamentais para o P2 era "Atentar para a topografia do comportamento - olhar nos olhos, expressão de interesse na conversa (comportamentos não verbais)" (p. 58), e pode-se verificar ser um objetivo necessário, visto que se comparado ao $\mathrm{P} 1$, ele apresentava menos de um terço de respostas em média. Contudo, observa-se que, do início para o desenvolvimento da terapia, a média de respostas não vocais de Gestos de Concordância $C$ se manteve estável para P2, mas elevou-se em cerca de $50 \%$ durante o encerramento do processo terapêutico, sendo um dado demonstrativo de melhora.

Fernandes (2012) encontrou como mais frequente para o cliente a categoria Relato, com 
média de 61,95\%. Pergher e Negrão (2012) também detectaram uma média alta para esta categoria, de $51 \%$. Neste trabalho, a categoria Relato apresentou médias menores, sendo a terceira categoria em frequência para P1, com médias em torno de $20 \%$, e a primeira para P2, com variação média de 25 a $37 \%$. Já em relação ao tempo de duração, para ambos os clientes o tempo médio ficou acima de $55 \%$, o que sugere que essa categoria prevalece sobre boa parte da sessão.

As categorias de Metas e Melhoras com baixa frequência neste trabalho também foram encontradas nestes patamares em outras pesquisas (Fernandes, 2012; Oshiro, 2011; Sadi, 2011). Contudo, é importante observar que tanto Metas quanto Melhora, apesar da baixa frequência, tiveram um tempo de exposição em ascensão, corroborando com os dados apresentados por Rocha (2012) sobre a melhora crescente dos clientes no decorrer da terapia, passando inclusive a formular metas e objetivos (tarefas de casa) durante as sessões.

Assim, apesar de serem clientes diferentes passando por um mesmo procedimento de intervenção, observa-se que a terapeuta apresentava um conjunto de práticas que se aplicavam para ambos os clientes. Esse conjunto de práticas puderam ser detectados pelas categorias comportamentais do SiMCCIT (Zamignani, 2007) e, em face do sucesso da terapia, pode-se relacionar a habilidades terapêuticas que possibilitaram sucesso no atendimento a clientes com transtorno de ansiedade social. Essas habilidades, descritas pela atuação da terapeuta, permitem concluir que a terapeuta procurou facilitar o relato do cliente durante toda a terapia (categorias de Facilitação e Gestos de Concordância T), Solicitava relato com frequência elevada, Informava com maior frequência e intensidade (t) no início da terapia e depois menos. Durante todo o procedimento, a terapeuta procurou Solicitar Reflexão, Interpretar, Recomendar e Aprovar com médias aproximadas.

Esses dados demonstram o importante papel do terapeuta durante o procedimento de intervenção, informando e estimulando o relato do cliente em sessão, proporcionando um ambiente acolhedor e que lhe favorecesse refletir sobre seu comportamento, produzindo autoconhecimento e, ao mesmo tempo, selecionava e modelava aspectos importantes de seu comportamento conforme as queixas e dificuldades identificadas.

O tempo de formação do terapeuta é discutido por Meyer (2009) como uma variável a ser considerada na interação terapêutica. Neste caso, apesar de ser considerada pouco experiente, apenas três anos de experiência profissional, a terapeuta que conduziu as sessões aqui analisadas se assemelhou aos terapeutas experientes, com mais de quinze anos de formação que, na descrição de Meyer (2009), apresentavam um padrão de interação mais complexo, com o terapeuta se utilizando de mais estratégias de intervenção.

Por sua vez, as categorias comportamentais do cliente podem funcionar como demonstração de preditores de bons resultados na terapia (Andrews, 2000; Castonguay et al., 2006; Silveira \& Kerbauy, 2000). Nesse caso, os clientes com transtorno de ansiedade social relataram (Relato) durante toda a terapia, com maior frequência e tempo no desenvolvimento e estabeleciam relações (Estabelece Relações) cada vez por mais tempo, o que sugere mais autoconhecimento com o desenvolvimento da terapia. Outra característica deste procedimento, assim como o detectado no estudo de Zamignani e Andery (2005), é que, para ambos os clientes, a terapia não apresentou comportamentos de oposição e discordância significativos. E apresentavam formulação de metas (Metas) e relato de melhora (Melhora) por maior período com o andamento das sessões.

Pesquisas que analisam a interação terapêutica, como apontam Ruiz-Sancho et al. (2013), auxiliam na compreensão do papel do terapeuta na psicoterapia. Neste sentido, este trabalho, na medida em que descreve as práticas do terapeuta no atendimento a clientes com transtorno de ansiedade social e descreve como esses clientes se comportam em psicoterapia, contribui para entender 
mudanças que ocorrem durante a sessão e, em consequência, melhorar a qualidade do suporte oferecido para indivíduos que procuram tratamento psicológico.

\section{Considerações finais}

Este estudo atingiu seus objetivos na medida em que, num primeiro momento, a categorização permitiu descrever os comportamentos do terapeuta e do cliente. Num segundo momento, a busca por padrões de ação do terapeuta e do cliente dentro da sessão permitiu descrever quais as práticas que um terapeuta comportamental se utiliza para alcançar os resultados da terapia, bem como quais os comportamentos do cliente demonstram efetivamente que os objetivos estejam sendo alcançados.

Novas pesquisas devem ser conduzidas, a fim de verificar a replicação dos resultados aqui descritos no atendimento a clientes com transtorno de ansiedade social. Novos estudos também devem ser conduzidos com clientes com outras queixas (diagnósticos), para se caracterizar o papel do terapeuta diante de cada população, bem como levantar dados que possam responder com maiores detalhes qual o papel do terapeuta neste processo, e como cada cliente pode influenciar na atuação do terapeuta. 
American Psychiatric Association (2002). DSM-IV-TR Manual de diagnóstico e estatística das pertubações mentais (C. Dornelles, Trans.). Porto Alegre: Artmed.

American Psychiatric Association (2013). Diagnostic and statistical manual of mental disorders (5th ed.). Washington, DC: Autor.

Andrews, H. B. (2000). The myth of the scientistpractitioner: A reply to R. King (1998) and N. King and Ollendick (1998). Australian Psychologist, 35, 60-63.

Barros, R. S. (2003). Uma introdução ao comportamento verbal. Revista Brasileira de Terapia Comportamental e Cognitiva, 5, 73-82.

Bolsoni-Silva, A. T. (2009). Habilidades sociais de universitários: procedimentos de intervenção na perspectiva da Análise do Comportamento. In R. C. Wielenska, (Eds.). Sobre comportamento e cognição: desafios, soluções e questionamentos. Santo André: ESETec Editores Associados, 21-52.

Castonguay, L. G., \& Beutler, L. E. (2006). Principles of therapeutics change that work. Oxford: Oxford University.

Castonguay, L. G., Constantino, M. J., \& Grosse, M. (2006). The working alliance: where are we and where should we go? Psychotherapy: Theory, Research, Practice, Training, 43(3), 271-279.

Fagundes, A. J. F. M. (1999). Descrição, definição e registro de comportamento. São Paulo, SP: Edicon.

Falcone, E. M. O. Fobia social (1995). In B. Rangé (Eds.). Psicoterapia comportamental e cognitiva (pp. 133-149). Campinas, SP: Psy.

Falcone, E. M. O., Gil, D. B., \& Ferreira, M. C. (2007). Um estudo comparativo da frequência de verbalização empática entre psicoterapeutas de diferentes abordagens teóricas. Estudos de Psicologia, 24(4), 451-461.

Fernandes, F. A. D. (2012). Relação terapêutica: uma análise dos comportamentos de terapeuta e cliente em sessões iniciais de terapia. Dissertação de Mestrado, Instituto de Psicologia, Universidade de São Paulo, São Paulo, SP.
Fonseca, R., Silva, P., \& Silva, R. (2007). Acordo inter-juízes: o caso do coeficiente kappa. Laboratório de Psicologia, 5(1), 81-90.

Kameyama, M. (2012). Intervenções sobre comportamentos de clientes que produzem sentimentos negativos no terapeuta. Dissertação de Mestrado, Instituto de Psicologia, Universidade de São Paulo, São Paulo, SP.

Kanamota, P. F. C. (2013). Estudo da influência das respostas de empatia e recomendação do terapeuta na interação terapeuta-cliente e descrição de efeitos de um procedimento de intervenção para o tratamento de mães de adolescentes com problemas de comportamento. Dissertação de Mestrado, Faculdade de Ciências, Universidade Estadual Paulista "Júlio de Mesquita Filho", Bauru, SP.

Kaplan, H. I., Sadock, B. J., \& Grebb, J. A. (1997). Fobia Específica e fobia social. In D. Batista (Trans.) Compêndio de psiquiatria: ciências do comportamento e psiquiatria clínica (pp. 562-567). Porto Alegre, RS: Artes Médicas.

Kazdin, A. E. (1982). Single-case research designs: methods for clinical and applied settings. Oxford: Oxford University Press.

Matos, M. A. (1991). As categorias formais de comportamento verbal de Skinner. In Anais da XXI Reunião Anual de Psicologia (pp. 333-341). Ribeirão Preto, São Paulo.

Meyer, S. B. (2001). A relação terapeuta-cliente é o principal meio de intervenção terapêutica? In H. J. Guilhardi, M. B. B. P. Madi, P. P. Queiroz, \& M. C. Scoz (Orgs.), Sobre comportamento e cognição: expondo a variabilidade (Vol.8, pp. 95-98). Santo André: ESETec.

Meyer, S. B. (2009). Análise de 'solicitação de informação' e 'recomendação' em banco de dados de terapias comportamentais. Tese de Livre-Docência, Instituto de Psicologia, Universidade de São Paulo,. São Paulo, SP.

Meyer, S. B., \& Vermes, J. S. (2001). Relação terapêutica. In Rangé, B. (Eds.) Psicoterapias cognitivo-comportamentais: um diálogo com a psiquiatria (pp. 101-110). Porto Alegre, RS: Artmed.

Oshiro, C. K. B. (2011). Delineamento experimental e caso único: a Psicoterapia Analítico Funcional com dois clientes 
difíceis. Tese de Doutorado, Universidade de São Paulo, São Paulo, SP.

Pergher, N. K., \& Negrão, L. F. (2012). Aplicação do sistema muldimensional na categorização de comportamentos na interação terapêutica ao acompanhamento terapêutico com foco no desenvolvimento de comportamentos pró-estudo. Cadernos de Pós-Graduação em Distúrbios do Desenvolvimento, 12(1), 88-97.

Rocha, J. F. (2012). Efeitos de uma intervenção comportamental com treino de habilidades sociais para universitários com fobia social. Dissertação de Mestrado, Faculdade de Ciências, Universidade Estadual Paulista "Júlio de Mesquita Filho", Bauru, SP.

Rossi, P. R. (2012). Categorização da quarta sessão de psicoterapias bem e mal sucedidas. Dissertação de Mestrado, Instituto de Psicologia, Universidade de São Paulo, São Paulo, SP.

Ruiz-Sancho, E. M., Frojan-Parga, M. X., \& Calero-Elvira, A. (2013). Functional analysis of the verbal interaction between psychologist and client during the therapeutic process. Behavior Modification 37(4), 516-542.

Sadi, H. M. (2011). Análise dos comportamentos de terapeuta e cliente em um caso de Transtorno de Personalidade Boderline. Tese de Doutorado, Instituto de Psicologia, Universidade de São Paulo, São Paulo, SP.

Silveira, F. F., Bolsoni-Silva, A. T., \& Meyer, S. B. (2010). Therapist's directive and nondirective behavior: analysis of their effects in a parent traininggroup. InternationalJournalofBehavioral Consultation and Therapy, 6(2), 124-133.

Silveira, J. M., \& Kerbauy, R. R. (2000). A interação terapeuta-cliente: uma investigação com base na queixa clínica. In R. R. Kerbauy
(Eds.). Sobre comportamento e cognição (pp. 213-221). Santo André, SP: Esetec.

Skinner, B. F. (1957). Verbal behavior. NewYork, NY: Appleton-Century-Crofts.

Tourinho, Z. E, Neno, S, Batista, J. R., Garcia, M. G., Brandão, G. G., Souza, L. M. ... Oliveira-Silva, M. (2007). Condições de treino e sistemas de categorização de verbalizações de terapeutas. Revista Brasileira de Terapia Comportamental e Cognitiva, 9(2), 317-336.

Xavier, R. N. (2011). Probabilidade de transição para o estudo da modelagem em dois estudos de caso de terapia analítico-comportamental infantil. Dissertação de Mestrado, Instituto de Psicologia, Universidade de São Paulo, São Paulo, SP.

Zamignani, D. R. (2007). Odesenvolvimento de um sistema multidimensional para a categorização de comportamentos na interação terapêutica. Tese de Doutorado, Instituto de Psicologia, Universidade de São Paulo. São Paulo, SP.

Zamignani, D. R., \& Andery, M. A. P. A. (2005). Interação entre terapeutas comportamentais e clientes diagnosticados com transtorno obsessivo-compulsivo. Psicologia: Teoria e Pesquisa, 21(1), 109-119.

Zamignani, D. R., \& Meyer, S. B. (2007). Comportamento verbal no contexto clínico: contribuições metodológicas a partir da análise do comportamento. Revista Brasileira de Terapia Comportamental e Cognitiva, 9(2), 241-259.

Zamignani, D. R., \& Meyer, S. B. (2011). Comportamentos verbais do terapeuta no sistema multidimensional para a categorização de comportamentos na interação terapêutica. Revista Perspectivas, 2(1), 25-45. 


\section{Vagner Angelo Garcia}

Mestre em Psicologia do Desenvolvimento e Aprendizagem pela Universidade Estadual Paulista Júlio de Mesquita Filho, São Paulo - SP. Brasil.

E-mail: vagner_asp@hotmail.com

\section{Alessandra Turini Bolsoni-Silva}

Docente da Universidade Estadual Paulista Júlio de Mesquita Filho, São Paulo - SP. Brasil.

E-mail: bolsoni@fc.unesp.br

\section{Glaucia Fernanda Galeazzi Nobile}

Mestranda em Psicologia do Desenvolvimento e Aprendizagem pela Universidade Estadual Paulista Júlio de Mesquita Filho, São Paulo - SP. Brasil.

E-mail: glau_fgn@hotmail.com

\section{Endereço para envio de correspondência:}

Universidade Estadual Paulista Júlio de Mesquita Filho. Engo. Luiz Edmundo C. Coube, s/n. CEP: 17033360. Bauru - SP. Brasil.

Recebido: 13/03/2014, Aprovado: 03/11/2015. 